\title{
Salud y educación de niños, niñas y adolescentes con TEA o TDI: una relación simbiótica para su garantía o vulneración. Caso Bucaramanga
}

\author{
Health and education of children and adolescents with ASD or IDD: a symbiotic relationship
} for their guarantee or infringes. Bucaramanga case

Saúde e educação de crianças, meninas e adolescentes com tea ou tdi: uma relação simbiótica para sua garantia ou vulneração. Caso Bucaramanga

DOI: https://doi.org/10.21803/penamer.13.26.424

Cirly Uribe Ochoa

https://orcid.org/0000-0002-5894-3795

Marien Correa Corredor

https://orcid.org/0000-0001-9942-664X

Ana Carolina Bernal Bueno

https://orcid.org/0000-0001-6823-4389.

\section{¿Cómo citar este artículo?}

Uribe, C., Correa, M. \& Bernal, A. (2020). Salud y educación de niños, niñas y adolescentes con TEA o TDI: una relación simbiótica para su garantía o vulneración. Caso Bucaramanga. Pensamiento Americano, 13(26), 113-126

DOI: https://doi.org/10.21803/penamer.13.26.424

\section{Resumen}

Este artículo es una reflexión sobre la relación simbiótica entre los derechos a la salud y a la educación de la población con Trastorno del Desarrollo Neurológico (TEA y TDI) en edad escolar, en el horizonte de lograr su inclusión. Para lo cual, se llevó a cabo una investigación de tipo cualitativa, a partir del análisis crítico del marco jurídico nacional e internacional que protege los derechos de las Personas con Discapacidad, las directrices del Ministerio de Educación Nacional sobre educación inclusiva y la información recabada a través de un trabajo de campo con familias de niños, niñas y adolescentes con estas condiciones, así como, la realización de entrevistas semi estructuradas a diferentes actores relacionados con la atención de esta población y la garantía de sus derechos, cuyo principal resultado es la constatación de las implicaciones que tiene para su escolarización y la no garantía acorde a sus necesidades del derecho a la salud.

Palabras clave: Personas con Discapacidad, Trastorno del desarrollo neurológico, derechos humanos, educación inclusiva.

\begin{abstract}
This paper is a reflection on the symbiotic relationship between the rights to health and education of the population with Neurological Development Disorder (TEA and TDI) in school age, on the horizon of achieving its inclusion. To this end, a qualitative research was carried out, based on a critical analysis of the national and international legal framework protecting the rights of persons with disabilities, the guidelines of the Ministry of Education on inclusive education and information gathered through field work with families of children and adolescents in these conditions, and the holding of semi structured interviews with different actors involved in the care of this population and the guarantee of their rights, whose the main result of which is the realization of the implications for their schooling and the lack of guarantees according to their needs of the right to health.
\end{abstract}

Keywords: Disabled people, Neurological development disorder, human rights and inclusive education.

\footnotetext{
*Adscrito al proyecto: intervención estratégica para la protección legal de los derechos a la salud y educación de la población de niños y adolescentes con trastorno del desarrollo neurológico (TDI, ASD) en la ciudad de Bucaramanga (Strategic intervention for the legal protection of the rights to health and education of the population of children and adolescents with neurological development disorder (TDI, ASD) in the city of Bucaramanga), investigación interinstitucional adelantada por docentes de las Universidades Cooperativa de Colombia (Bucaramanga) y Universidad de Santander (Bucaramanga). Convocatoria CONADI. 2018.
} 


\section{Resumo}

Este artigo é uma reflexão sobre a relação simbiótica entre os direitos à saúde e à educação da população com Transtornos do Desenvolvimento Neurológico (TEA e DDI) em idade escolar, no horizonte de alcançar sua inclusão. Para isso, foi realizada uma pesquisa qualitativa, com base na análise crítica do arcabouço jurídico nacional e internacional que tutela os direitos das pessoas com deficiência, nas diretrizes do Ministério da Educação Nacional sobre educação inclusiva e nas informações coletadas em trabalhos de campo. com famílias de crianças e adolescentes nessas condições, bem como a realização de entrevistas semiestruturadas com diferentes atores relacionados ao cuidado dessa população e à garantia de seus direitos, cujo principal resultado é a verificação das implicações que isso tem para os seus. escolaridade e a não garantia de acordo com suas necessidades do direito à saúde.

Palavras chave: Pessoas com Deficiência, Transtorno do Neurodesenvolvimento, Direitos Humanos, Educação Inclusiva.

\section{Perfiles}

Licenciada en Educación Básica con énfasis en Ciencias Sociales, Estudiante de Doctorado en Educación y Sociedad, Universidad de La Salle. Bogotá, Colombia. Docente investigadora de la Facultad de Derecho - Universidad Cooperativa de Colombia, sede Bucaramanga. Dirección electrónica: cirly.uribeo@campusucc.edu.co.

Abogada, Magister en Relaciones Internacionales, Universidad del Salvador. Buenos Aires, Argentina. Líder del grupo de investigación Los Mediadores de la Facultad de Derecho- Universidad Cooperativa de Colombia, seccional Bucaramanga. Dirección electrónica: marien.correa@ucc.edu.co.

Abogada, Estudiante de Doctorado en Ciencias Políticas y Jurídicas en la Universidad Pablo de Olavide, Sevilla, España. Magister en Hermenéutica Jurídica y Derecho. Docente investigadora de la Facultad de Derecho y Ciencias Políticas de la Universidad Libre. Dirección electrónica: anac.bernalb@unilibre.edu.co.

\section{Cirly Uribe Ochoa}

Marien Correa Corredor

Ana Carolina Bernal Bueno 


\section{Introducción}

Las Personas con Discapacidad (en adelante PcD) representan a nivel mundial un alto porcentaje poblacional, según cifras de la Organización Mundial de la Salud (OMS, 2011) "Más de mil millones de personas viven en todo el mundo con alguna forma de discapacidad" (p.xi). Pese a ello, su visibilización es reciente; pues desde la antigüedad se les ha marginado, violentado, cosificado y negado, especialmente a quienes tenían discapacidad cognitiva o mental, empezando por el ámbito familiar en el que a muchos se les escondíal. En este contexto, aquellos han tenido que recorrer un largo camino para lograr la positivación de sus derechos, lo cual es reciente, dado que, se halla sustancialmente en el marco de la Declaración Universal de los Derechos Humanos, promulgada por la Organización Internacional de las Naciones Unidas (ONU), en 1948, cuyo Artículo primero reza: "Toda persona tiene todos los derechos y libertades proclamados en esta Declaración, sin distinción alguna de raza, color, sexo, idioma, religión, opinión política o de cualquier otra índole, origen nacional o social, posición económica, nacimiento o cualquier otra condición”. Sin embargo, aunque hay avances, la situación de las PcD en el mundo sigue siendo precaria, por decir lo menos. Así lo reconoce la misma OMS (2011) al afirmar que "En todo el mundo, las personas con discapacidad tienen peores resultados sanitarios, peores resultados académicos, una menor participación económica y unas tasas de pobreza más altas que las personas sin discapacidad” (p. 5).

En Colombia este panorama no es distinto y se complica más dado que, "no [se] tiene una cifra exacta de las personas con discapacidad" (Minsalud, 2018, p. 2). Sin embargo, "Desde el año 2.002 (...) se han identificado y caracterizado 1.404 .108 personas" (ibid.), con categorías sobre el tipo de alteración, clasificadas como: el movimiento del cuerpo, manos, brazos, piernas; el sistema nervioso; los ojos; el sistema cardiorrespiratorio y las defensas; los oídos; la voz y el habla; la digestión, el metabolismo y las hormonas; el sistema genital y reproductivo; la piel; y los demás órganos de los sentidos (ibid., p. 19). Ello hace imposible identificar en cuál de éstas se ubica a las personas con Trastorno del Espectro Autista (en adelante TEA) y Trastorno del Desarrollo Intelectual (en adelante TDI); no para rotularlas sino para establecer la información que permita dar cuenta de sus condiciones de salud o de vida en general, así como de las barreras que les impide hacer parte de la sociedad.

En consecuencia, la información estadística que se halla es generalizada. Por ejemplo, se encuentra que: "De cada 100 personas con discapacidad mayores de 24 años al momento del registro: 42 tenían como último nivel educativo aprobado la Primaria, 20 la Secundaria, y 31 personas no habían alcanzado ningún nivel educativo"; adicionando el dato de "32\% [que] no sabe leer y escribir" (ibid., p. 13). Otras cifras respecto a salud indican:

El 71,53\% de la población con discapacidad registrada a nivel nacional se encuentra afiliada al Sistema General de Seguridad Social en Salud (SGSSS), de ellas, el 69,27\% están afiliadas al régimen subsidiado, $30,26 \%$ al contributivo y un $0,4 \%$ al régimen de excepción. (Minsalud, 2014,p. 15)

No se niega la validez de la información, porque además permite vislumbrar altos niveles de exclusión y de vulneración de derechos. Pero no permite conocer las realidades particulares de quienes tienen TEA o TDI, ni la de sus familias. Así lo reconocen diferentes organizaciones que aglutinan personas con esta clase de discapacidad, como la Liga Colombiana de Autismo (LICA) (Jerez, 2019); por lo que es posible asumir que, al menos en Colombia, se conoce poco sobre la realidad que vive la población con alguna tipología de Trastorno del Desarrollo Neurológico.

En tal sentido, todo esfuerzo por identificar condiciones de vida que permitan visibilizar el contexto de quienes tienen TEA o TDI, permitirá trabajar ha-

1 Incluso, aun algunas familias en la época actual esconden a sus parientes cuando presentan cierta clase de condiciones de discapacidad.

Pensamiento Americano Vol. 13 - No. 26 - p.p. 113-126 • 2020 • Julio-Diciembre • Corporación Universitaria Americana • Barranquilla, Colombia • ISSN: 2027-2448 http://publicaciones.americana.edu.co/index.php/pensamientoamericano/index 
cia el restablecimiento de derechos y al proceso de su inclusión social. Este es el fin último de la investigación realizada por la Universidad Cooperativa de Colombia, campus Bucaramanga y la Universidad de Santander, en el periodo 2018 - 2019, para responder a la pregunta: ¿Cuál puede ser la intervención estratégica de protección jurídica de los derechos a la salud y a la educación, de la población de niños, niñas y adolescentes con Trastorno del Desarrollo Neurológico (TDI y TEA) en la ciudad de Bucaramanga?

Un trabajo académico realizado a partir del análisis documental del marco jurídico nacional e internacional que protege los derechos de las $\mathrm{PcD}$, como el deber ser que garantiza el respeto y la valoración de su dignidad humana. Confrontado con el trabajo de campo realizado a través de la aplicación de una encuesta a familias con niños, niñas y adolescentes (en adelante NNA) con TDI y TEA; un análisis de archivos que configuran las historias jurídicas del proceso de exigibilidad que han realizado sus familias en procura del restablecimiento de los derechos a la salud y a la educación; y entrevistas semi estructuradas a servidores públicos de las instancias municipales de salud y educación, responsables de la garantía de tales derechos, así como a especialistas sobre los Trastornos del Desarrollo Neurológico, en los que se enmarcan las dos condiciones de la población sujeto de la investigación. "La contemplación, la reflexión y la comunicación no son disciplinas, sino más bien máquinas que construyen universales". (Wenger, 2016). Todo ello, desde el marco teórico del modelo biopsicosocial con enfoque de derechos.

\section{La discapacidad desde tres enfoques: Médico, social y psicosocial}

A través de la historia, las sociedades han abordado la realidad de las discapacidades de distintas maneras. Desde concebirlas como un castigo divino, embrujo o maleficio, hasta su comprensión a partir de referentes científicos.

En tal sentido, durante muchos años prevaleció el modelo médico en el que se conciben las discapacidades como enfermedades, precisándose la rehabilitación de quienes las tienen. "En este modelo la persona con discapacidad requiere de cuidados clínicos prestados en forma de tratamiento individual, encaminado a conseguir la cura o una mejora del sujeto, o un cambio en él, concentrándose en las consecuencias de la enfermedad" (Hernández Ríos, 2015, p. 48), pero imperando el sentido de ser personas con menor valor que las demás. Un enfoque que asume la perspectiva de un cuerpo dañado en una persona aislada de su contexto, como si no tuviera influencia en éste. Argumentando la "concepción médica rehabilitadora de la discapacidad que se centra en el individuo para mejorar sus deficiencias o la psicológica que se centra en la mejora del proceso de adaptación” (Verdugo Alfonso, 2001, p. 2).

Para la década de los años 70 se planteó por el Médico Psiquiatra Engel (1977) una perspectiva biopsicosocial de la discapacidad. Entendió que el modelo médico o biomédico no era suficiente para asumir el caso de quien tenía alguna tipología de discapacidad. Debía tenerse en cuenta "el contexto social en el cual él vive y el sistema complementario ideado por la sociedad para tratar los efectos disruptivos de las enfermedades" (p. 132). Por ello, el fin del modelo biopsicosocial propuesto "es determinado por la función histórica del médico para determinar si la persona que está solicitando ayuda está enferma o está bien” (ibid.)

emana el modelo social, que concibe a la discapacidad en relación con el entorno social en el que los individuos se desenvuelven, asignando a dicho contexto el paradigma de la exclusión; son las barreras socioculturales las que dificultan o impiden que PcD se integren a la vida colectiva. Por ello "subraya el papel que la discriminación institucional y el prejuicio desempeñan en moldear la experiencia de discapacidad" (Verdugo Alfonso, 2001, p. 4). Indica este autor, citando a Olkin (1999), que: "Los seguidores del modelo [social] piensan que la discapacidad es un constructo social, entendiendo por esto que su significado es mejor comprendido en términos de

Pensamiento Americano Vol. 13 - No. 26 - p.p. 113-126 • 2020 • Julio-Diciembre · Corporación Universitaria Americana • Barranquilla, Colombia • ISSN: 2027-2448 http://publicaciones.americana.edu.co/index.php/pensamientoamericano/index 
cómo la sociedad percibe a la discapacidad” (ibid.).

Por lo anterior y teniendo en cuenta que, cada uno de los modelos con los cuales se ha abordado la discapacidad tiene fortalezas y limitaciones, en las últimas décadas se propuso "integrar perspectivas tanto médicas como psicológicas y sociales" (ibid. p. 10) para asumir la discapacidad o mejor las discapacidades de manera integral o multidimensional, como es la vida humana. Así:

La discapacidad está definida como el resultado de una compleja relación entre la condición de salud de una persona y sus factores personales, y los factores externos que representan las circunstancias en las que vive esa persona. A causa de esta relación, los distintos ambientes pueden tener efectos distintos en un individuo con una condición de salud. Un entorno con barreras, o sin facilitadores, restringirá el desempeño/ realización del individuo; mientras que otros entornos que sean más facilitadores pueden incrementarlo. La sociedad puede dificultar el desempeño/realización de un individuo tanto porque cree barreras (ej. edificios inaccesibles) o porque no proporcione elementos facilitadores (ej. baja disponibilidad de dispositivos de ayuda). (OMS, 2001, p. 27)

Es decir, se reasume la corriente de Engel con su Modelo Biopsicosocial, porque además de las condiciones físicas, fisiológicas, mentales o cognitivas impuestas por una enfermedad, lesión o condición genética, ha de sumárseles aspectos actitudinales de la persona misma en condición de discapacidad (cuando le es posible); esto es, en términos generales, el cómo asume su realidad. Así mismo, se incluyen los criterios imperantes en la sociedad respecto a la discapacidad, desde las actitudes individuales y colectivas de inclusión o exclusión, las que a su vez suelen ser tácitas o explícitas y que se traducen en aspectos identificables. Por ejemplo, las de exclusión se encuentran en barreras actitudinales, arquitectónicas, comunicacionales, entre otras, que dificultan e imposibilitan la participación de las $\mathrm{PcD}$ en la vida social, política, económica y cultural de su entorno; todo lo cual sumado, violenta la igualdad de oportunidades a la que tienen derecho y además determina las condiciones y la calidad de vida, haciendo que ésta sea mucho más difícil de lo que impone la misma discapacidad.

En tal sentido es que la OMS (2001) determina los factores contextuales que integran la vida de cualquier individuo, compuestos a su vez por factores ambientales y factores personales. Los primeros comprenden:

(...) al mundo físico natural con todas sus características, el mundo físico creado por el hombre, las demás personas con las que se establecen o asumen diferentes relaciones o papeles, las actitudes y valores, los servicios y sistemas sociales y políticos, y las reglas y leyes. (p. 207)

Por su parte, los factores personales son aquellos "que tienen que ver con el individuo como la edad, el sexo, el nivel social, experiencias vitales" (ibid.) y que impactan de manera positiva o negativa la vida de cualquier persona, entre ellas quienes tiene discapacidad.

En consecuencia, este modelo resulta de mayor pertinencia para abordar la discapacidad, por cuanto configura la inclusión como un lenguaje y una acción para trascender las diferencias y eliminar situaciones de discriminación en el marco de los derechos humanos. Por ende, en los últimos años se habla del enfoque de derechos al reconocer que las PcD son titulares de derechos reconocidos constitucionalmente, como expresión de su dignidad humana y, por tanto, corresponde al Estado garantizarlos de manera integral y a la sociedad contribuir a la erradicación de toda forma de discriminación.

En la teoría del enfoque de derechos se reconoce a la persona con discapacidad en razón de su dignidad humana, por lo tanto, el concepto de discapacidad se concreta en la Convención de Derechos Humanos de las Personas con Disca- 
pacidad como una respuesta teórica y normativa, en la que confluyen los modelos médico y social. En esta Convención la discapacidad se concibe como un problema dentro de la sociedad y no como una característica de la persona, por lo tanto, no es una situación que tiene efectos estrictamente particulares, sino que es una cuestión que concierne derechos humanos. (Hernández Ríos, 2015, p. 56)

Así que, el llamado es a reconocer y exigir que se reconozca como sujeto de derechos a quien tiene una discapacidad. Los Estados, garantizando su ejercicio; la sociedad, respetando al otro con sus diferencias y eliminando actitudes hostiles y discriminatorias, con apertura de oportunidades en diferentes ámbitos sin condiciones de desigualdad, porque éstas representan escenarios de exclusión social. Bajo este enfoque, no debe haber lugar a restricciones para el ejercicio de derechos fundamentales como lo son, entre otros, la salud y la educación.

\section{Resultados de la investigación}

Teniendo en cuenta que la investigación se situó desde la concepción dada por el modelo biopsicosocial con enfoque de derechos frente a las discapacidades, los resultados se ubican en los factores ambientales y personales que afectan las condiciones de niños, niñas y adolescentes con TEA y TDI en la ciudad de Bucaramanga.

La primera precisión que se debe hacer es que el trastorno del espectro autista y el trastorno del desarrollo intelectual son condiciones y no enfermedades. Es decir, dado el actual desarrollo científico se comprende que son para toda la vida y, por consiguiente, no hay curación. Pero, en procura del mayor grado de adaptabilidad, funcionalidad y autonomía que sea posible alcanzar, para lograr su integración en la vida familiar y social, existen procesos terapéuticos, apoyos y ciertos medicamentos (en el caso del autismo).

Los resultados de las encuestas aplicadas a la muestra tomada evidenciaron mayor porcentaje de varones entre NNA con TEA, a diferencia de quienes tienen TDI en l os que el género no fue relevante.

De otra parte, no se hallaron NNA con mayores niveles de autonomía, toda vez que su dependencia es total del cuidador, quien en la mayoría de los casos suele ser la madre o en su defecto, las abuelas.

En cuanto a dificultades manifestadas por las familias, se puso de presente su situación económica. La pobreza es un factor común en gran parte de esta población, cuyos ingresos, en su mayoría, no superan los dos salarios mínimos legales vigentes. Se constató también con datos del Observatorio Digital Municipal de Bucaramanga, donde se indica que el 93\% de la población con discapacidad registrada corresponde a estratos socioeconómicos 1,2 y 3 , en los que prevalece la precariedad económica y los bajos niveles de bienestar en todos o casi todos los indicadores, tales como: ingresos, salud, vivienda, educación, recreación, entre otros. La OMS y el Banco Mundial (2011) confirman que es un fenómeno universal:

Las personas con discapacidad presentan tasas más altas de pobreza que las personas sin discapacidad. En promedio, las personas con discapacidad y las familias con un miembro con discapacidad tienen mayores tasas de privaciones -como inseguridad alimentaria, condiciones deficientes de vivienda, falta de acceso a agua potable y salubridad, y acceso deficiente a atención de salud y poseen menos bienes que las personas y familias sin una discapacidad. (p. 12)

Esta realidad de carencias socio económicas de las familias de las PcD configuran un ambiente que contribuye a la vulneración de los derechos de esta población, puesto que, discapacidades como las relacionadas con el Trastorno del Desarrollo Neurológico constituyen una gran carga económica y emocional para las familias, máxime cuando no cuentan con ingresos suficientes, esencialmente porque, en general, solo uno de los cónyuges puede

Pensamiento Americano Vol. 13 - No. 26 - p.p. 113-126 • 2020 • Julio-Diciembre · Corporación Universitaria Americana $\cdot$ Barranquilla, Colombia $\cdot$ ISSN: 2027-2448 $\mathrm{http} / / /$ publicaciones.americana.edu.co/index.php/pensamientoamericano/index 
trabajar, dado que el otro, quien habitualmente es la madre, debe dedicar todo su tiempo ( 24 horas al día, siete días a la semana) a cuidar de su hijo con discapacidad. Esto, en el mejor de los casos; porque en la información recabada se halló alto porcentaje de madres solas con sus hijos, lo que hace más difícil la situación, puesto que, deben soportar toda la responsabilidad económica y emocional de responder en su totalidad por el bienestar de su hijo con discapacidad y de sus otros hijos (cuando los hay).

Se debe agregar que, los altos costos relacionados con gastos de transporte hacen más gravosa la situación de estas familias. Como se dijo antes, dado que los NNA con TEA y TDI carecen de autonomía, dependen totalmente de una persona adulta para desplazarse a cualquier diligencia, sea ésta de salud o relacionada con sus procesos educativos (cuando los hay). Además, si su condición es TEA, la mayoría no tolera el ruido y el tumulto casi siempre presente en el transporte público, por lo que a los cuidadores les implica trasladarse en taxi, elevándose los costos que, junto a los demás gastos que deben cubrir por la condición de su hijo/a, impacta negativamente los ingresos familiares.

Definitivamente, las barreras que viven los NNA con TEA y/o TDI y sus familias, se convierten en tropiezos para facilitar la calidad de vida de todos. Las familias y las mujeres en particular viven estados de preocupación y angustia al encontrarse con esos obstáculos en su día a día. Una de estas circunstancias adversas es la misma familia extensa, pues la búsqueda de un culpable es frecuente, así como de preguntas sin respuestas sobre el por qué su hijo/a tiene tal condición.

Algunos casos dejaron entrever vestigios del viejo modelo de la prescindencia, en los que se intenta negar la existencia del hijo o se acusa a la madre de ser la responsable, porque no se deseaba que aquel tuviera ese trastorno y no se acepta, o se vive con un complejo de culpa permanente por las situaciones adversas a las que se ven obligadas. Sin embargo, hubo familias que aceptaron más rápido a su hijo con su trastorno, sin negar que vivieron "el duelo", como lo reconoció una de las madres entrevistadas.
Otra barrera, y no menos impactante, es el sistema de salud dentro del cual deben realizar un proceso largo, desgastante e insolidario. La primera falla del sistema de salud colombiano está en la dificultad para acceder a exámenes especializados que permitan un diagnóstico real y oportuno en el primer año de vida del niño o niña, principalmente cuando se trata de TEA. Un médico neuro pediatra entrevistado manifestó que, entre más temprana la detección, mejor la posibilidad de realizar tratamientos que faciliten la adaptación, explicando que ésta se debe hacer antes de los dos años. Infortunadamente, como contestaron las madres encuestadas, el Programa de Crecimiento y Desarrollo del sistema de salud pocas veces indaga por el neurodesarrollo de los bebés; y aunque fueron ellas quienes detectaron las primeras señales de las condiciones de autismo en sus hijos o sus maestros en la escuela en sus primeros años, hubo reticencia por parte de los profesionales de la salud para ordenar los exámenes que se requerían. Solo en pocos casos, cuando se contaba con algún recurso económico, los exámenes se realizaron a cargo de los padres o madres. Por ello, el 67\% de los NNA que formaron parte de la muestra, no tuvieron diagnóstico oportuno.

Ya hay muchos, muchos estudios, que han demostrado en el mundo, que si yo empiezo la intervención terapéutica rehabilitadora antes de los dos años versus con los que empezaron la intervención con el diagnóstico después de dos años, el grado de funcionalidad futura del primer grupo del que fue intervenido de forma temprana, va a ser muy superior del que fue intervenido después de los dos años. (Villamizar, entrevista realizada en 2017).

El doctor Villamizar (2017) hizo saber que el diagnóstico tardío se debe a que, "algunas IPS., limitan la capacidad diagnóstica que puede tener un médico especialista, les dicen: no puedes formular tantos exámenes, eso hace que algunos especialistas trabajen con lo básico, pero eso finalmente puede ahorrarle dinero a las EPS”. Por ello, los diagnósticos tempranos no son la regla sino la excepción. 
Pero no solo es la falta de diagnósticos oportunos lo que se censura, sino el no cubrimiento de todo lo que implica el servicio de salud además de tratamientos de habilitación, rehabilitación y conexos con su condición neurológica y genética, tales como "Pañales, transportes, medicamentos, citas con especialistas"; "Alimentación y suplementos nutricionales"; "Instrumentos para las terapias"; entre otros. Exigencias que, al ser cubiertas por las familias, implican un gasto del $40 \%$ de sus ingresos, dada la negativa de las EPS.

Como una situación adicional, hay NNA que tienen varias (dos o tres) condiciones de discapacidad además de TEA o TDI, como: "Síndrome convulsivo"; "Retardo Psicomotor"; "Síndrome prader Willi"; "Síndrome de Angelman"; "Hidrocefalia"; "Trastorno del desarrollo Motor"; "Lesión zona lumbar de la columna"; "Disminución de los miembros inferiores"; "Microcefalia”; "Baja visión”; "Síndrome de Jouberth"; "Hipoxia cerebral”; "Atención Dispersa"; entre otras. Muchas de ellas, incluyendo al autismo, son consideradas enfermedades huérfanas, generalmente de alto costo económico y emocional para los cuidadores.

La cuestión es que, con una o varias condiciones, el acceso a la salud de estos NNA que requieren medicamentos, terapias, transporte y todo aquello conexo, no siempre se obtiene como respuesta a la garantía de un derecho fundamental. Por ello, el $86 \%$ de las familias encuestadas ha tenido que recurrir a la acción de tutela, lo cual se convierte en mayor carga sensible y desgaste físico para las familias que deben estar entre juzgados, EPS e IPS, no siempre con resultado satisfactorio porque aun teniendo fallo a favor no se cumple el mismo y deben acudir al incidente de desacato. El 14\% que no acudió a la rama judicial fue desconocimiento de la existencia de la acción de tutela y menos del desacato.

Las situaciones planteadas en el párrafo anterior dejan en evidencia el no cumplimiento de la normatividad nacional e internacional existente para la protección del derecho a la salud de las $\mathrm{PcD}$ y en particular de los NNA. En consecuencia, si su derecho a la salud se vulnera se les está coartando la posibilidad de habilitaciones o rehabilitaciones, según corresponda, lo cual incidirá negativamente en el acceso a la escolarización en aulas regulares como lo plantea la educación inclusiva. Es decir, surge otra barrera que madres y padres de NNA con TEA y TDI se ven obligados a sortear.

Hasta hace menos de 20 años en Colombia, los NNA con discapacidad visual, auditiva o cognitiva (esencialmente) solo podían educarse en centros especializados de carácter privado, mayoritariamente, con maestros formados en educación especial, o en colegios convencionales, pero en aulas diferentes a las que estudiaba el resto de alumnos catalogados “normales". Ello, aun cuando desde 1994 se empieza a hablar de educación inclusiva sustentada en la Declaración de Salamanca (Walker, 2013, p. 14), referida nuevamente en la Convención sobre los Derechos de las Personas con Discapacidad y su Protocolo Facultativo, aprobados en diciembre de 2006 por las Naciones Unidas, en la que concita a los Estados parte a que reconozcan:

El derecho de las personas con discapacidad a la educación. Con miras a hacer efectivo este derecho sin discriminación y sobre la base de la igualdad de oportunidades, los Estados Parte asegurarán un sistema de educación inclusivo a todos los niveles, así como la enseñanza a lo largo de la vida. (Art.24)

Planteando además que, para tal fin deben brindar "a las personas con discapacidad la posibilidad de aprender habilidades para la vida y desarrollo social, a fin de propiciar su participación plena y en igualdad de condiciones en la educación y como miembros de la comunidad" (ibid.).

La Convención referida fue aprobada por el Estado colombiano en el año 2009 con la Ley 1346. Pero fue hasta el 2013 que en Colombia se expidió la Ley Estatutaria 1618 "Por medio de la cual se establecen las disposiciones para garantizar el pleno ejercicio

Pensamiento Americano Vol. 13 - No. 26 - p.p. 113-126 • 2020 • Julio-Diciembre • Corporación Universitaria Americana • Barranquilla, Colombia • ISSN: 2027-2448 $\mathrm{http} / / /$ publicaciones.americana.edu.co/index.php/pensamientoamericano/index 
de los derechos de las personas con discapacidad" y en el 2017, mediante el Decreto 1421, se reglamentó la atención educativa a las PcD en el marco de la educación inclusiva. Así, se institucionaliza el derecho a la educación en aulas regulares y en todos los niveles, para los niños, niñas, adolescentes, jóvenes y adultos con discapacidad.

Similarmente, ya había habido un impulso de política pública en perspectiva de la inclusión educativa, denominada Revolución Educativa e implementada a partir del 2002. "La inclusión de la población con discapacidades en la escuela común está en el corazón” (MEN, 2007, p. 2). Por ello, se planteaba que la inclusión significaba:

Atender con calidad, pertinencia y equidad a las necesidades comunes y específicas que estas poblaciones presentan. Para lograrlo ha sido necesario que gradualmente el sistema educativo defina y aplique concepciones éticas que permitan considerar la inclusión como un asunto de derechos y de valores, lo que está significando implementar estrategias de enseñanza flexibles e innovadoras que abren el camino a una educación que reconoce estilos de aprendizaje y capacidades diferentes entre los estudiantes y que, en consonancia, ofrece diferentes alternativas de acceso al conocimiento y evalúa diferentes niveles de competencia. (ibid., p. 4)

Sin embargo, al tiempo presente la garantía del derecho a la educación para niños, niñas y adolescentes con TEA y/o TDI se ve limitada, pese a la consigna de las últimas décadas que pretende inclusión real y no solo nominal de las personas con discapacidad, cuando se lee: "todos los y las estudiantes cuentan, y cuentan por igual” (negrilla en texto original) (UNESCO, 2017, p. 12)-

Así que, el marco jurídico, las directrices nominales, las declaraciones de las organizaciones suprana- cionales y los intentos desde diferentes focos de atención, frente a la inclusión en aulas regulares de NNA con discapacidad, en particular TDI y TEA, no han impedido que sigan subsistiendo barreras para lograr el acceso, la permanencia y el egreso exitoso de sus procesos de escolarización. En el Estado social de derecho impera una visión teleológica, en donde las normas responden a una finalidad social y constitucional (Romero \& Ibarra, 2017). La política que se viene difundiendo tropieza con restricciones presupuestales de las instituciones educativas, que les impide realizar ajustes razonables relacionados con aspectos arquitectónicos de planta física, cualificación del personal docente y administrativo, y consecución de apoyos pedagógicos y metodológicos acordes con los diagnósticos de los estudiantes incluidos; todo lo cual tiene costos que los establecimientos educativos oficiales no están en capacidad de sufragar, a pesar de la obligación legal de recibir PcD.

De modo que, la realidad difiere del deber ser sobre todo frente a quienes tienen Trastorno del Espectro Autista, porque si bien es cierto la Secretaría de Educación de Bucaramanga, en el caso en estudio, y otras dependencias similares en otras ciudades y departamentos establecen unas directrices sustentadas en las normas vigentes, a aquellos se les niega su inclusión a la educación aduciendo diversas razones: falta de cupos, inexistencia de apoyos pedagógicos ${ }^{2} \mathrm{o}$ condicionando su ingreso a que éste tenga tutor sombra $^{3} \mathrm{o}$ alguno de sus padres le acompañe durante la jornada, lo cual supone una carga mayor para los padres, quienes deben multiplicar sus esfuerzos entre la atención escolar de su hijo y su trabajo.

De las entrevistas realizadas a rectores y funcionarios públicos se dedujo que, el grupo que mayor resistencia tiene frente a la inclusión de NNA con discapacidad, son los profesores.

Los docentes llegan a ser la población más resistente a los procesos de inclusión, debido a las

2 Estos son personales de apoyo especialistas en educación especial (la mayoría) que, ayudan al profesor titular del curso, con el proceso de planeación y manejo de los niños con discapacidad.

3 Persona que acompaña al NNA., durante la jornada escolar y ayuda a reforzar el proceso de aprendizaje y a controlar/manejar los comportamientos que puedan afectarlo y o afectar a los demás.

Pensamiento Americano Vol. 13 - No. 26 - p.p. 113-126 • 2020 · Julio-Diciembre • Corporación Universitaria Americana • Barranquilla, Colombia • ISSN: 2027-2448 $\mathrm{http} / / /$ publicaciones.americana.edu.co/index.php/pensamientoamericano/index 
demandas de trabajo y requerimiento de los entes; si le llevas un niño con discapacidad a un docente porque le falta uno, dice que no porque le va a generar un problema; lo ven como una carga, como un problema, como un castigo. (Rector 1-Colegio público-, entrevista 2018.)

Es un paradigma complejo que, como se señaló en páginas anteriores, corresponde a las barreras socioculturales del imaginario consolidadas por la falta de competencias pedagógicas frente a la inclusión social. En varias instituciones educativas se encontraron profesores que no son licenciados sino titulados en otras profesiones, como economistas, administradores de empresas, por dar algunos ejemplos, pero vinculados a la planta docente por resultados de concursos.

La actuación de los docentes está sujeta a las características particulares del contexto en el que tiene lugar y tendrá que sacar aprovecho [sic] de sus herramientas cognoscitivas para actuar con asertividad. Este hecho marca diferencias en la práctica educativa y abre un abanico de posibilidades para la atención a la diversidad, reconociendo que el aprendizaje es una construcción sociocultural, y que éste será significativo en la medida que se interactúa con los escenarios de la cultura, los propios de la educación y el trabajo colaborativo en interacción con otros pares. (Correa, Bedoya y Alzate, 2015, p. 51)

Lo cierto es que, en estos escenarios existe discrepancia de criterios respecto a la clase de educación que deben recibir los escolares con discapacidad, aunque con otras visiones de parte de aquellos, también pueden ser agentes transformadores.

Para unos, deben estar en aulas convencionales a fin de que aprendan más si comparten con quienes no tienen discapacidad, proveyendo a éstos últimos la oportunidad de cultivar valores relacionados con el respeto por las diferencias (Cortes, 2019, citada por Delgado, 2019). Para otros, el proceso de enseñanza-aprendizaje no fluirá correctamente por el mismo ambiente de la escuela; por ejemplo, el número de estudiantes por curso hace que el manejo sea más complejo y no se pueda responder dentro de los parámetros exigidos en la educación inclusiva. Se dijo en una de las entrevistas: "Yo estoy en el colegio.... y allá tengo grupos hasta de 45 estudiantes; entonces cuando hay un grupo de 45 , si hay un niño incluido eso dificulta muchísimo más la inclusión” (Apoyo pedagógico, comunicación personal, realizada el 27 de septiembre de 2018). Agregando además que, el tiempo que se les dedica no es de calidad, mucho menos cuando estos estudiantes tienen necesidades especiales:

Se les pierde mucho tiempo al incluirlos, porque ellos tienen unos potenciales muy singulares, entonces cuando un niño es excepcional o cuando un niño tiene menor capacidad (...) se le ponen esas actividades básicas, pero no cien por ciento, sino unos tiempitos reducidos, entonces digamos que se les está dejando de potenciar esas habilidades que tienen en aras de atender a todo un grupo con el tema mayor. (Apoyo pedagógico, ibid.)

Habría que decir también que, las instituciones educativas no cuentan con condiciones estructurales de recursos y talento humano para brindar una atención integral a los estudiantes con discapacidad. Luego, el reconocimiento del derecho a ser incluido que exige cambio de paradigmas en toda la sociedad también requiere inversión económica.

Ellos debían estar insertos con los otros que tuvieran normalidad, eso seguramente tuvo su sustento y ha sido bien para muchas cosas, pero mal para otras, porque una cosa es lo que diga la ley que tengan derechos y todo, y otra cosa es que las instituciones estén preparadas en su parte humana, en su parte de infraestructura, en su parte social, para atender a esa población” (Rector 1 colegio público, comunicación personal realizada el 27 de septiembre de 2018).

Dentro de los aspectos a mejorar, se planteó en for- 
ma coincidente la inversión en el talento humano y en infraestructura física y tecnológica; pero, igualmente, se requiere mayor compromiso de las entidades prestadoras del servicio de salud (Colegio, Cote Uribe. Comunicación escrita enviada el marzo 5 de 2019).

A todo lo anterior ha de sumársele un entorno que todavía transita entre la hostilidad y la indiferencia. Cerrar la puerta de la educación a NNA con discapacidad o no garantizar su calidad, son actos de exclusión que transgreden el ingreso al trabajo y la participación política y social, entre otros. La educación hace ciudadanos, visibiliza a las personas, las hace miembros del mundo común, en un "estar juntos y los unos con los otros de los diversos" (Arendt, 2018, p. 5) respetando la otredad. Eso sí, para "que la educación tenga este papel de 'derecho capacitador', debe ser de gran calidad, equitativamente disponible, pensado para acabar con la discriminación y para fomentar que cada niño dé los frutos que su talento e intereses le permitan" (Walker, 2013, p. 4).

Las PcD existen y tienen necesidades como los demás e incluso, más que los demás. La cuestión es que, las mismas políticas públicas implementadas las incluye en la categoría de población vulnerable y sus ejecutores olvidan que son sujetos con derechos. Entonces, algunos programas suelen tener corte asistencialista que no cubre a toda la población, ni resuelve estructuralmente las condiciones de pobreza y discriminación. Su inclusión social requiere programas articulados con presupuesto suficiente.

Todos aquellos factores en el entorno de una persona que, cuando están presentes o ausentes, mejoran el funcionamiento y reducen la discapacidad. Entre ellos se incluyen aspectos tales como que el ambiente físico sea accesible, la disponibilidad de tecnología asistencial adecuada, las actitudes positivas de la población respecto a la discapacidad, y también los servicios, sistemas y políticas que intenten aumentar la participación de las personas con una condición de salud en todas las áreas de la vida. También la ausencia de un factor puede ser un facilitador, por ejemplo, la ausencia de estigma o actitudes negativas entre la población (OMS, 2001, p. 208).

En concordancia, es indispensable una nueva actitud de los gestores a cargo de diferentes servicios públicos: funcionarios de las EPS e IPS a cargo de la atención en salud y profesores comprometidos con la educación de los colombianos. Eso sí, haciéndolos partícipes de los diseños de los programas.

Concebir al docente como gestor de la transformación fue pertinente porque es el empoderado de las prácticas educativas y pedagógicas. Por eso, es un error adelantar transformaciones sin él como líder y aliado. Adicionalmente se requiere el trabajo colectivo y compromiso de la comunidad educativa para instaurar la gestión escolar con calidad y equidad, que responda por las necesidades de formación y educación de la diversidad de la población (subraya fuera de texto). (Correa, Bedoya y Alzate, 2015, p. 49)

Es decir, la inclusión de NNA con TEA y TDI en todos los escenarios sociales requiere la articulación familia-sociedad-Estado ${ }^{4}$ y consta de un marco normativo: Constitución Política de Colombia (Arts. 13, 44, 45, 47 y 49); Ley 361, 1997; Ley 1098,2006; Ley 1306; 2009; Ley 1346,2009; Ley 1752, 2015; y Ley Estatutaria 1618, 2013; Decreto 2082, 1996; Decreto 19, 2012; y Decreto 1421 de 2017, entre otros. También, con decisiones jurisprudenciales garantes de los derechos de esta población: T-518, 2006; T-1222,2008; C-930,2009; T-045, 2013; T-847;2013; y T-557, 2016; sin perjuicio de otros pronunciamientos. Ordenamiento adecuado a los instrumentos internacionales sobre protección de personas con discapacidad.

Esta omisión en la responsabilidad de contribuir a la inclusión en salud y en educación de niños, niñas y adolescentes con Trastorno del Espectro Autista o

\footnotetext{
4 Cumpliendo el postulado de la corresponsabilidad que predica el artículo 44 de la Constitución Política de Colombia. Por ello, se requiere compromiso de todos los implicados incluyendo a las familias, para que comprendan que solo bajo colaboración efectiva se garantiza el respeto al derecho a la salud y a la educación.
}

Pensamiento Americano Vol. 13 - No. 26 - p.p. 113-126 • 2020 • Julio-Diciembre · Corporación Universitaria Americana $\cdot$ Barranquilla, Colombia $\cdot$ ISSN: 2027-2448 $\mathrm{http} / / /$ publicaciones.americana.edu.co/index.php/pensamientoamericano/index 
con Trastorno de Desarrollo Intelectual, además de constituir vulneración de derechos fundamentales, contribuye a la victimización de dicho colectivo y de los cuidadores, iniciando por una omisión de diagnósticos tempranos que permitan prever acciones oportunas para mejorar las condiciones de vida de estos niños; continuando con su desescolarización a pesar de las políticas de educación inclusiva; e incidiendo en su futuro ejercicio laboral, político y sociocultural. Entonces, ¿dónde queda el papel del Estado?

Como sea la relación de ciudadanos y estado: libertad y política permanecen separadas en lo decisivo y ser libre en el sentido de una actividad positiva, que se despliega libremente, queda ubicado en el ámbito de la vida y la propiedad, donde de lo que se trata no es de nada común sino de cosas en su mayoría muy particulares (Arendt, 2018, p. 51).

El fenómeno de la sucesiva negligencia hallada en la investigación, marca necesidades sobre cómo erradicar orientaciones ajenas al modelo biopsicosocial en enfoque de derechos.

\section{Conclusiones}

La inserción de niños, niñas y adolescentes con discapacidad en la sociedad, en particular al sistema educativo, exige la previa garantía plena del derecho fundamental a la salud mediante la atención médica y el suministro de lo necesario para su habilitación. En tal sentido, incluirlos en aulas convencionales no se puede reducir a confinar cuerpos en un salón y con ello cumplir con la "formalidad" ante el Ministerio de Educación Nacional.

Si Colombia es un Estado Social de Derecho asumido como nación multicultural que respeta y valora la diversidad, debe reconocerse a la educación inclusiva en su dimensión ontológica a partir de la correlación de la acción de educar con el proceso de aprender de distintas maneras, ritmos y niveles, entendiendo que los seres humanos, con o sin discapacidad, tienen diferencias en sus rasgos de desarrollo y su capacidad de abstracción y razonamiento. Disi- militudes que pueden generar cuestionamientos entre los profesores e incluso entre los padres y madres de familia sobre qué pueden aprender los NNA con discapacidad cognitiva; qué se les puede o debe enseñar; para qué se escolarizan; entre otras.

Más allá de esos interrogantes, la escuela enseña sobre el mundo desde su dimensión natural y su dimensión cultural, entendida ésta como el mundo inventado por los seres humanos donde se requieren conocimientos que ayuden a adaptarse al entorno y luego transformarlo; pues la presencia humana siempre transforma el entorno. Esta intencionalidad de la educación es válida para todos los seres humanos, independientemente de cualquier condición. Por consiguiente, hay más por aprender de las personas con discapacidad; ellos enseñan al resto de sociedad a salir de las zonas de confort; enseñan a amar, comprender y entender al otro; y ayudan a la sociedad a desarrollar la alteridad, la solidaridad y la empatía. Es decir, tienen mucho para dar.

Como corolario se indica que, el Estado no debe seguir cohonestando el no cumplimiento del ordenamiento jurídico y en particular el desconocimiento de los derechos fundamentales a la salud y a la educación cuya titularidad está en cabeza de NNA con TEA y TDI, por parte de las empresas prestadoras de salud; de los funcionarios públicos responsables de la educación; de los directivos de instituciones educativas; de profesores; $y$, algunas veces, sus propios padres y/o madres.

Además, no se puede continuar con un discurso sin acciones a favor de la vida antes de nacer, porque el negar la salud luego de nacido, principalmente a personas cuyas familias no cuentan con los recursos económicos y las redes de apoyo para hacer que la vida de sus hijos sea digna, con posteriores restricciones para acceder a la educación, es una evidencia del desconocimiento de unos derechos también incorporados en la Declaración Universal de los Derechos Humanos. 


\section{Referencias}

Arendt, H. (2018). ¿Qué es la política? - Fragmento l. Introducción a la política II - Fragmento 3B. ¿Qué es la política? Comprensión y política (1 ${ }^{\mathrm{a}}$ ed.), 5-8; 27-61. Ciudad de México: Partido de la Revolución Democrática. Obtenido de https://www.prd. org.mx/libros/documentos/libros/Politica-Hannah.pdf

Asamblea General de las Naciones Unidas. (1948). Declaración Universal de los Derechos Humanos. 217 (III) A. Paris.

Congreso de la República. (27 de febrero de 2013). Ley Estatutaria de los derechos de las personas con discapacidad. [Ley $1618 \mathrm{de}$ 2013]. DO: 46.446

Congreso de la República. (3 de junio de 2015). Modifica Ley 1482 de 2011 para sancionar penalmente discriminación contra personas con discapacidad. [Ley 1752 de 2015]. DO: 49.531

Congreso de la República. (31 de julio de 2009) Aprueba Convención sobre los Derechos de las personas con Discapacidad. [Ley 1346 de 2009]. DO: 47.427.

Congreso de la República. (5 de junio de 2009). Protección de Personas con Discapacidad Mental y Régimen de Representación Legal de Incapaces Emancipados. [Ley 1306 de 2009]. DO: 47.371.

Congreso de la República. (7 de febrero de 1997). (1997) Ley de integración social de las personas con limitación. [Ley 361 de 1997]. DO: 42.978.

Congreso de la República. (8 de noviembre de 2006). Código de la Infancia y la Adolescencia. [Ley 1098 de 2006]. DO: 48.717.

Constitución Política de Colombia [Const.]. (1991). Artículos 13, 44, 45, 47 y 49 [Título II]. Ed. 2016. Obtenido en https:// www.corteconstitucional.gov.co/inicio/Constitucion\%20 politica\%20de\%20Colombia.pdf

Correa, J., Bedoya Sierra, M., \& Agudelo Alzate, G. (2015). Formación de docentes participantes en el programa de educación inclusiva con calidad en Colombia. Revista latinoamericana de educación inclusiva, 9(1), 43-60. Obtenido en https://dialnet.unirioja.es/servlet/articulo?codigo $=5155476$
Corte Constitucional, Sala Sexta de Revisión. (26 de noviembre de 2013). Sentencia T-847, 2013. [MP Nilson Pinilla Pinilla].

Corte Constitucional, Sala Cuarta de Revisión. (1 de febrero de 2013). Sentencia T-045, 2013. [MP Gabriel Eduardo Mendoza Martelo].

Corte Constitucional, Sala Octava de Revisión. (13 de octubre de 2016). Sentencia T-557, 2016. [MP Alberto Rojas Ríos].

Corte Constitucional, Sala Plena. (10 diciembre de 2009). Sentencia C-930, 2009. [MP Jorge Ignacio Pretelt Chaljub].

Corte Constitucional, Sala Quinta de Revisión. (5 de diciembre de 2008). Sentencia T-1222, 2008. [MP Rodrigo Escobar Gil].

Corte Constitucional, Sala Sexta de Revisión. (7 de julio de 2006). Sentencia T-518, 2006. [MP Marco Gerardo Monroy Cabra].

Delgado, C. (4 de junio de 2019).¿Educación inclusiva en Bucaramanga? Vanguardia Liberal. Obtenido en https://www. vanguardia.com/area-metropolitana/bucaramanga/educacion-inclusiva-en-bucaramanga-CA1031488

Engel, G. (1977). The Need for a New Medical Model: A Challenge for Biomedicine. Science, New Series, 196 (4286), 129-136.

Hernández Ríos, M. I. (2015). El Concepto de Discapacidad: De la Enfermedad al Enfoque de Derechos. Revista CES Derecho 6(2), 46-49. Obtenido en http://www.scielo.org.co/pdf/cesd/ v6n2/v6n2a04.pdf

Jerez, A. C. (1 de abril de 2019). Autimo: un diagnóstico que no es una etiqueta. El Tiempo. Obtenido en https://www.eltiempo.com/vida/salud/panorama-del-autismo-en-colombia-344162

Ministerio de Educación Nacional [MEN] (septiembre-diciembre 2007). Educación para todos. Al Tablero, 43. Obtenido en https://www.mineducacion.gov.co/162l/article-141881.html

Ministerio de Salud y Protección Social [Minsalud]. (junio de 2014). Línea base Observatorio Nacional de Discapacidad. Análisis descriptivo de indicadores. Observatorio Nacional de Discapacidad. Obtenido en https://discapacidadcolombia.

Pensamiento Americano Vol. 13 - No. 26 - p.p. 113-126 • 2020 • Julio-Diciembre • Corporación Universitaria Americana • Barranquilla, Colombia • ISSN: $2027-2448$ http://publicaciones.americana.edu.co/index.php/pensamientoamericano/index 
com/phocadownloadpap/ESTADISTICAS/L\%C3\%ADnea\%20Base\%20Discapacidad\%20OND.pdf

Ministerio de salud y protección social. [Minsalud] (2018). Sala situacional de discapacidad de las Personas con Discapacidad (PCD). Obtenido en https://www.minsalud.gov.co/sites/rid/ Lists/BibliotecaDigital/RIDE/DE/PS/sala-situacional-discapacidad-junio-2018.pdf

Organización de las Naciones Unidas para la Educación, la Ciencia y la Cultura [UNESCO] (2017). Guía para asegurar la inclusión y la equidad en la educación. Obtenido de http:// down21-chile.cl/cont/cont/2017/336_2_guia_para_asegurar_la_inclusion_y_la_equidad_en_la_educacion.pdf

Organizacion Mundial de la Salud [OMS]. (2001). Clasificación Internacional del Funcionamiento, de la Discapacidad y de la Salud: CIF. Obtenido de http://www.imserso.es/InterPresent2/groups/imserso/documents/binario/435cif.pdf

Organización Mundial de la Salud y Banco Mundial [OMS - BM] (2011). Resumen Informe Mundial sobre Discapacidad. 27. Obtenido de https://www.who.int/disabilities/world report/2011/summary_es.pdf

Presidencia de la República (29 de agosto de 2017). Regulación educación inclusiva. [Decreto 1421 de 2017]. DO: 50.340.

Presidencia de la República. (10 de enero de 2012). Decreto Antitrámites. [Decreto 19 de 2012]. DO: 48.308.

Presidencia de la República. (18 de noviembre de 1996). Atención educativa para personas con limitaciones o con capacidades o talentos excepcionales. [Decreto 2082 de 1996]. DO: 42.922.

Romero Marín, L. C., \& Ibarra Lozano, J. E. (2017). La pensión especial anticipada de vejez: Un análisis desde la perspectiva de la teoría de la eficacia simbólica del derecho. Advocatus, 2(29), 41-57.

Verdugo Alfonso, M. Á. (2001). La concepción de la discapacidad en los modelos sociales (mesa redonda). Universidad de Murcia. Obtenido en https://www.um.es/discatif/TEORIA/Verdugo-ModelosSoc.pdf
Walker, J. (2013). Igualdad de derechos. Igualdad de oportunidades. La educación inclusiva para niños con discapacidad. Campaña Mundial por la Educación - Handicap International, 1-36. Obtenido en http://www.campaignforeducation. org/docs/reports/Equal\%20Right\%20Equal\%20Opportunity\%20ES.pdf

Wenger Calvo, R. (2016). El cine como "imagen del pensamiento" según el constructivismo filosófico de G. Deleuze. Amauta, 14(27), 119-134. https://doi.org/10.15648/am.27.2016.9
2020, Vol. 13(26) 113-126. (CThe Author(s) 2020 Reprints and permission: www.americana.edu.co http://publicaciones.americana.edu.co/index.php/pensamientoamericano/index 